\title{
A chacun son père
}

\author{
Jean-Loup CLEMENT
}

Hôpital Édouard-Herriot, Lyon

\begin{abstract}
RÉSUMÉ
L'Insémination Artificielle avec Donneur (IAD) est pratiquée, en France, depuis 35 ans, comme méthode palliative de la stérilité masculine. Elle a permis la naissance d'environ quarante-cinq mille enfants. L'évaluation de cette population de parents et de leurs enfants est difficile à réaliser. Elle est néanmoins possible.
\end{abstract}

II ressort d'une étude approfondie que les enfants, devenus adultes, sont globalement satisfaits et que l'anonymat du don de sperme est la meilleure façon d'établir une paternité et de constituer une famille.

Mot clés : stérilité, paternité, insémination avec donneur, anonymat

\section{INTRODUCTION}

Lorsque j'ai commencé à travailler au CECOS (Centre d'Étude et de Conservation des CEufs et du Sperme humains) de Lyon, en 1978, comme psychologue, la méthode de l'Insémination Artificielle avec Donneur (IAD) était en pleine élaboration : tout était matière à réflexion et à débat car il s'agissait de rendre officielle et transparente une méthode servant à pallier la stérilité masculine. La priorité était d'arriver à satisfaire les couples qui se présentaient au CECOS : permettre à des hommes stériles de devenir pères. Surtout dans ces années-là, les hommes et les femmes attendaient depuis longtemps, certains ayant été informés tardivement de la possibilité de recours à l'IAD.

Vingt-cinq ans plus tard, j'ai effectué une recherche sur les enfants conçus par IAD [1]. L'objectif était alors de recueillir l'avis de ces enfants, devenus adultes, sur la méthode de procréation artificielle et de ses conséquences pour eux- mêmes, et d'analyser leurs réactions. J'avais imaginé que des enfants profiteraient de cette occasion pour témoigner de leurs difficultés et de leur agressivité contre le système de la conception par IAD et la politique des CECOS. Cela n'a pas été le cas.

Cette recherche a apporté et confirmé plusieurs enseignements :

1. Le seul nombre qui peut être avancé est celui de quarantecinq mille enfants conçus par IAD à partir du service des CECOS.

2. Il est impossible de savoir quel pourcentage de ces enfants sont informés de leur mode de conception. Des évaluations sont réalisées auprès des futurs parents au moment de leur demande d'IAD, mais cela reste le recueil d'une intention : il y a en effet une grande distance entre une intention exprimée à un moment précis et sa réalisation des années plus tard, d'autant plus dans le domaine de l'intime comme celui de la génération et l'éducation des enfants.

3. Il est impossible de savoir si les enfants qui ont connaissance de leur mode de conception sont satisfaits ou non du système de l'anonymat du don. Ceux que j'ai rencontrés, au nombre de vingt et un, pour ma recherche étaient globalement satisfaits. Des enfants, sans pouvoir les dénombrer, ont tenté ou tentent d'obtenir des renseignements sur le donneur. Les CECOS n'étant pas submergés par de telles demandes, il est possible de penser que ces enfants sont très peu nombreux.

4. Dans le domaine de la génération et de la filiation particulièrement, il est toujours difficile d'opérer la part de la réalité de celle du fantasme. C'est ainsi qu'un adulte, conçu par IAD, peut énoncer quasiment dans le même

\section{Correspondance :}

Dr Jean-Loup CLEMENT - Hôpital Édouard-Herriot, place

d'Arsonval, 69003 Lyon - Tel 0472110160 .

Email<jean-loup.clement@sante.univ-lyon1.fr 
temps son souhait de connaître le donneur, puis de demander seulement une photographie et pour finalement ne rien demander du tout. Rien n'est jamais acquis. II est difficile de prendre une décision. Tout est assez mouvant et tant mieux.

Dans le processus de l'IAD, il y a plusieurs protagonistes :

a) le couple receveur, un homme stérile et sa partenaire ;

b) le donneur et son conjoint, c'est-à-dire un père, son ou ses enfant(s) et en principe son conjoint, s'il n'en est pas séparé;

c) le médecin et tous les praticiens qui garantissent le système de I'IAD.

Et en arrière-fond, les magistrats qui ont produit des jugements sur la paternité IAD et in fine le législateur.

\section{LA DEFINITION DE LA PATERNITE}

La pratique médicale officielle de l'IAD existe depuis plus de trente ans et elle peut se pérenniser tant qu'il existe un consensus sur la définition de la paternité.

La paternité repose d'une part sur un apport biologique et génétique, ce qui en constitue le patrimoine génétique, et d'autre part sur la prise en charge affective et matérielle de l'enfant, la contribution à son éducation, c'est la paternité symbolique où l'adulte masculin se définit comme père, pour qu'ensuite l'enfant le reconnaisse comme tel.

Sur la paternité biologique : il n'existe pas de preuve objective de cet apport. Cette paternité est toujours présumée, jusqu'à preuve du contraire. C'est la femme qui nomme le père en tant qu'il a été son partenaire dans la relation sexuelle : celle où l'enfant a été conçu. Dans la plupart des cas, l'homme reconnaît implicitement qu'il est le géniteur de l'enfant et construit son histoire de père à partir de la naissance de l'enfant.

Il est des cas où l'homme conteste la paternité de son enfant. C'est-à-dire que l'homme ne veut pas assumer l'éducation d'un enfant et tente de prouver qu'il n'est pas biologiquement son géniteur. Avant que les typages HLA soient reconnus comme fiables, le désaveu de paternité était prouvé par des témoignages, comme celui de l'absence physique de l'homme pendant la période présumée de la conception. Souvent, lorsque la mère en était d'accord, le désaveu était recevable d'autant plus qu'un autre homme, qui se définissait comme l'amant, était présent pour assumer la paternité de l'enfant. C'est donc à nouveau un système de nomination d'un homme pour être père.

Dans d'autres cas, un homme fait une reconnaissance de paternité naturelle pour un enfant dont il n'est pas le géniteur car il a connu la femme postérieurement à la naissance de l'enfant. Il déclare vouloir se comporter en père. Les tribunaux reconnaissent que cet homme peut être déclaré père de l'enfant. Cela se complique lorsque ce même homme demande l'annulation de la paternité, lorsqu'il se sépare de la mère de l'enfant. II est reconnu alors que la paternité n'était pas complète vu qu'il manque la dimension biologique de géniteur. Et a contrario, en ayant étudié des jugements de tribunaux civils, j'ai pu lire que des magistrats faisaient remarquer, pour justifier leur refus d'annulation de la paternité, qu'il était préjudiciable à l'enfant de changer de père autant de fois que leur mère a changé d'amants !

À l'inverse, on reconnaît officiellement que des adultes hommes et femmes, hommes ou femmes soient parents sans être les géniteurs de l'enfant. C'est ainsi que l'on confie des enfants abandonnés par leurs géniteurs à d'autres adultes qui se portent garants pour en être les parents. Une fois le jugement du tribunal prononcé, il n'est plus possible de se rétracter.

La paternité se décline suivant des situations différentes. C'est ainsi qu'il devient possible d'élaborer la notion de paternité IAD en posant un pré-requis : un homme est stérile, du sperme fécondant est substitué, par l'intermédiaire du CECOS et du médecin, et inséminé à la femme. On définit alors que l'absence de lien biologique du père à l'enfant est officielle : la mère ayant seule un lien biologique et génétique. C'est le schéma de l'adoption, mais unilatéralement. Le don d'ovocytes quelques années après l'IAD est venu confirmer qu'il était possible qu'un enfant soit issu unilatéralement du père seulement, la mère n'ayant pas donné son patrimoine génétique.

La paternité par IAD est une construction de l'institution médicale. C'est un modèle élaboré pour tenter de pallier la stérilité masculine. Les hommes et les femmes choisissent I'IAD par défaut, pour ne pas dire par dépit ! En l'absence de thérapeutique de toutes les stérilités masculines, c'est le recours à l'IAD qui se présente comme une des solutions.

\section{LE ROLE DU DONNEUR}

Dès le début des CECOS, Georges David, en tant que fondateur, s'est appliqué à rendre positif le geste du donneur : tenter qu'il n'y ait pas de rétribution objective et pécuniaire au don de sperme. L'analogie avec la pratique du don du sang a permis la confirmation que le don de cellules humaines n'est pas rétribué. Même si on ne peut pas placer sur le même plan, les motivations du don de gamètes avec celles du don du sang, l'histoire du don du sang a facilité le fait de ne pas rechercher un paiement pour le don de sperme, alors que c'était le cas dans les pratiques clandestines et privées. Avec les CECOS, l'officialisation de l'IAD dans des hôpitaux publics a pu édicter des règles sur la non-rémunération du don et l'anonymat des donneurs. Mais il a été beaucoup reproché au CECOS de ne pas avoir suffisamment de considération pour le donneur et de le percevoir comme étant seulement capable de produire du sperme fécondant !

De 1987 à 1994, les médecins qui ont pratiqué le don d'ovocytes ont complètement brouillé les cartes sur la question des conditions du don de gamètes. Le don intrafamilial d'une femme (mère) à sa propre sœur a été un pratique médicale répandue. Le bénéfice était pour une femme déjà mère de permettre à sa sœur d'accéder à la maternité, via les services d'un médecin. Or, il est important de rappeler que l'on ne fait pas d'enfant en famille ni même avec les alliés, comme l'enseigne l'ethnologue Françoise Héritier [3]. C'est la règle de l'exogamie qui régit la génération : chaque personne quitte son groupe d'origine pour s'allier avec une autre d'un groupe 
différent et ainsi faire des enfants. Pour le don de gamètes, la loi de 1994 a servi à confirmer cette dimension. Les donneurs sont et doivent être toujours des étrangers.

En ce qui concerne le don de sperme, le bénéfice est de l'ordre de l'intime, c'est-à-dire qu'il y a une dimension érotique de l'acte de masturbation. La rationalisation des propos de venir en aide à des couples stériles connus ou inconnus autorise le don. C'est le discours manifeste de la solidarité. Ensuite, il faut bien pouvoir expliquer la pénurie constante de donneurs de sperme. Donner son sperme contribue à la conception et à la naissance d'enfants en dehors de son propre couple. Quand on interroge des hommes sur les motifs de refus d'être donneur de sperme, c'est toujours sur l'argument de savoir que des enfants pourraient exister dans des familles autres que la leur qui est l'explication de ce même refus. À ce sujet, les travaux de recherche ethnologique enseignent sur la question de la rétribution du don d'une personne à une autre dans le cadre de l'organisation de groupes sociaux [4]. Longtemps, il a été recherché un "contredon» au don de sperme, ce dernier est dans l'intime et ne peut pas revêtir une forme matérielle. Le bénéfice est dans l'ordre du fantasme. Un homme est sollicité et il répond à cette sollicitation pour permettre de concevoir des enfants. Et c'est pourquoi le don de sperme doit être anonyme pour protéger cet homme et son entourage de la conséquence réelle de son acte. Le donneur n'est pas une abstraction. II lui est demandé d'être père. C'est un homme qui exerce (en principe) dans l'actualité sa paternité avec la mère de ses enfants. II est important de ne pas l'isoler et de ne pas le dénommer par le vocable "le donneur». C'est un homme qui a une histoire et ses enfants sont en mesure d'en témoigner.

II y a quelques années, je posai la question, lors d'un enseignement d'épistémologie à des étudiants de deuxième année de Sciences, de savoir quelles seraient leurs réactions s'ils apprenaient que leurs parents ont été donneurs de gamètes [2]. J'ai retenu entre autres réactions celles du fantasme d'inceste entre enfants : c'est-à-dire la rencontre entre ceux nés dans la famille et ceux nés à la suite du don de leur parent. Et aussi, la réaction sur le fait de n'avoir pas été informé "plus tôt» de ce don. C'est toujours le fantasme des enfants sur la sexualité de leurs parents et le fait d'être obligé d'accepter les frères et sœurs qui viennent après eux. Ces réactions m'amènent toujours à rappeler que ce sont les adultes qui font les enfants et que ces derniers n'en sont que la résultante. Les hommes et les femmes n'informent pas leur enfant au moment où ils donnent du sperme ou des ovocytes.

\section{DON ANONYME OU IDENTIFIABLE}

Toutes les réactions des enfants conçus par IAD, celles de leurs parents, des donneurs confirment ma conviction que le don doit être anonyme pour le confort psychologique de tous. Si le don n'est pas anonyme et que l'identité du donneur est divulguée à la majorité de l'enfant, il y a des conséquences. Je prends comme exemple le plus récent, celui du RoyaumeUni, qui a décidé que le don de gamètes n'était plus anonyme à partir de l'année 2005 : les enfants conçus à partir de cette date pourront à leur majorité connaître le donneur, c'est-à-dire à partir de l'année 2023.
Ce système est d'emblée bancal. Les parents doivent élever leur enfant avec cette perspective qu'il y a un homme/tiers dans le couple qui a eu un rôle précis et qui l'a encore dixhuit ans après la naissance de l'enfant. C'est l'image de l'adultère puisque des enfants sont identifiés dans des familles différentes : ces enfants étant reliés à un homme précis, le donneur qui n'est pas un géniteur. Cette image de l'adultère pèse sur le couple qui a eu recours à l'IAD. II faut que le couple qui élève l'enfant puisse penser que la conception de leur enfant provient d'un donneur, certes ; mais la reconnaissance et la dette se règlent dans l'imaginaire : pour le père d'abord et avant tout, la mère et ensuite l'enfant.

La proposition de faire coexister deux systèmes : l'un anonyme et l'autre identifiable procède d'une confusion dans la définition du don de gamètes, de la paternité et de la maternité dans le contexte d'infertilité. Demander aux couples demandeurs d'un don de gamètes de choisir entre un don anonyme ou un don personnalisé est sadique. Ces couples, fragilisés par la situation d'infertilité, ne peuvent pas se déterminer de façon sereine.

Je pense qu'il ne faut pas mettre les adultes dans une situation proche du dilemme. S'ils choisissent le don anonyme, leur enfant pourra leur reprocher ne pas avoir choisi le don identifiable. S'ils choisissent le don identifiable, leur enfant pourra leur reprocher de ne pas avoir choisi le don anonyme. Le couple parental peut aussi changer d'avis en cours d'histoire. Donc je suis favorable pour qu'un seul modèle existe, celui de l'anonymat total depuis le début de la conception. En plus, dans le système où l'anonymat du donneur est levé, ce n'est qu'à la majorité de l'enfant. Je pense qu'il est inhumain de demander à des hommes et des femmes de s'engager dix-huit ans à l'avance sur la question de l'éducation de l'enfant, la façon de gérer la filiation, etc... La rétractation est impossible alors qu'il existe ce droit de rétractation. C'est le principe de la liberté individuelle qui est mise à mal.

\section{CONCLUSION}

II est difficile de s'accorder sur le système le plus adéquat de la pratique médicale du don de gamètes. Je pense qu'il faut privilégier l'histoire de la paternité et de la maternité qui est à construire avec l'enfant qui a été conçu avec un artifice de fécondation, par impossibilité d'une conception autre : priorité aux parents. L'enfant a le droit de connaître ses origines génétiques, c'est-àdire sa genèse, au sens de l'histoire personnelle qui a prévalu dans sa conception et sa venue au monde. L'identité biologique et sociale du donneur ou de la donneuse est annexe.

\section{RÉFÉRENCES}

1. CLEMENT J.-L. : Mon père, c'est mon père : l'histoire singulière des enfants conçus par Insémination Artificielle avec Donneur. Paris, L'Harmattan, 2006.

2. CLEMENT J.-L. : Quand passent les cigognes... Dialogue, 1998, 142 : 82-94.

3. HERITIER F. : Les deux sœurs et leur mère. Odile Jacob, 1994.

4. MAUSS M. : Essai sur le don (1924). Paris, P.U.F., Ed. Quadrige, 2007. 
Manuscrit reçu : janvier 2008 ; accepté février 2008.

Communication présentée lors du XXIVème Congrès de la SALF, Colmar décembre 2007.

\author{
ABSTRACT \\ One father for everybody \\ Jean-Loup CLEMENT
}

Donor insemination (DI) has been performed for 35 years in France as a palliative method for male sterility. About forty five thousand children have been born after DI. Evaluation of this population of parents and children is difficult for ethical and administrative reasons.

However, a qualitative study can be performed under particular conditions. DI children, now adults, were able to express their personal experience. Gamete donation is anonymous in France. This measure is contested by some, but approved by others. The donor often has his own family and cannot be expected to be recognized by children that he did not raise and only after the age of 18. Parents of DI children prefer to remain the only parent (one father and one mother) for their children. Children prefer the donor to remain imaginary without denying his role in conception.

CECOS centres are not overwhelmed by untimely demands from children looking for the donor. The actual system seems to be the best for everyone.

Key Words : paternity, donor insemination, anonymity, children 